\title{
Norm attaining bilinear forms on $C^{*}$-algebras
}

\author{
by \\ J. Alaminos, R. Payá and A. R. Villena (Granada)
}

\begin{abstract}
We give a sufficient condition on a $C^{*}$-algebra to ensure that every weakly compact operator into an arbitrary Banach space can be approximated by norm attaining operators and that every continuous bilinear form can be approximated by norm attaining bilinear forms. Moreover we prove that the class of $C^{*}$-algebras satisfying this condition includes the group $C^{*}$-algebras of compact groups.
\end{abstract}

1. Introduction and statement of main results. It is a famous theorem by E. Bishop and R. Phelps that the norm attaining linear functionals on a Banach space $X$ are dense in the dual space $X^{*}$. The situation becomes completely different if we ask for the density of the norm attaining bilinear forms in the space of all continuous bilinear forms on $X$. In fact, there are Banach spaces for which this density fails to be true $[1,8,13]$ and quite a lot of attention has been paid to the question of finding Banach spaces $X$ for which every continuous bilinear form can be approximated by norm attaining bilinear forms $\phi$ on $X$ (i.e. there exist $x, y \in X$ such that $\|x\|=\|y\|=1$ and $\|\phi\|=\phi(x, y))[5,9,11]$. Recently it was shown in [4] that every continuous bilinear form on a commutative $C^{*}$-algebra $A$ can be approximated by norm attaining bilinear forms on $A$. The first step in order to prove that result was to show that every weakly compact operator from $A$ into an arbitrary Banach space can be approximated by norm attaining operators. This latter result strongly relies on the proof of W. Schachermayer [16] that every weakly compact operator from the real $C(K)$ for a compact Hausdorff space $K$ into a Banach space can be approximated by norm attaining operators. It should be noted that every continuous bilinear form on a Banach space $X$ can be identified with a continuous linear operator from $X$ into $X^{*}$. Moreover if the bilinear form is norm attaining, then so is the corresponding operator, but the converse is far from true [11]. Of course the question of the density of norm attaining operators between Banach spaces

2000 Mathematics Subject Classification: 47C15.

The first and second authors are supported by MCYT Grant BFM2000-1467.

The third author is supported by MCYT Grant PB1998-1358. 
is the natural extension of the question of the density of norm attaining linear functionals on Banach spaces, which is answered by the Bishop-Phelps Theorem.

In this paper we are concerned with the extension of [4, Theorems 2 and 3] and [16, Theorem B] to noncommutative $C^{*}$-algebras. Our approach heavily rests on the pattern introduced in [16] and subsequently used in [4]. The basic principles underlying those results can be stated as follows.

Theorem 1. Let $X$ be a Banach space. Suppose that for every weakly compact subset $W$ of $X^{*}$, every $x_{0}^{*} \in X^{*}$, and every $\varepsilon>0$ there is a continuous linear operator $S: X^{*} \rightarrow X^{*}$ with the following properties:

(i) $\|S\|=1$,

(ii) there is $x_{0} \in X$ with $\left\|x_{0}\right\|=1$ and $\left(S x_{0}^{*}\right)\left(x_{0}\right)=\left\|x_{0}^{*}\right\|$,

(iii) $\left\|S x^{*}-x^{*}\right\|<\varepsilon$ for each $x^{*} \in W$.

Then every weakly compact operator from $X$ into an arbitrary Banach space can be approximated by norm attaining continuous linear operators.

If in addition every continuous linear operator from $X$ into $X^{*}$ is weakly compact, then every continuous bilinear form on $X$ can be approximated by norm attaining continuous bilinear forms.

In order to put into action this principle in the noncommutative context, we have to put a restriction on the $C^{*}$-algebra.

Theorem 2. Let $A$ be a $C^{*}$-algebra satisfying the following hypothesis:

$(\mathrm{H}) \quad$ For every positive linear functional $\phi$ on $A$ and every $\varepsilon>0$ there exist a positive linear functional $\psi$ on $A$ and $\delta>0$ with the property that if $p \in A^{* *}$ is a projection with $\psi(p)<\delta$ then there exists a central projection $z \in A^{* *}$ such that $z p=0$ and $\phi(\mathbf{1}-z)<\varepsilon$.

Then every weakly compact operator from $A$ into an arbitrary Banach space can be approximated by norm attaining operators and every continuous bilinear form on $A$ can be approximated by norm attaining bilinear forms on $A$.

In the last section we will show that the class of $C^{*}$-algebras appearing in Theorem 2 includes the $C^{*}$-algebras of the form $\bigoplus_{i \in I} M_{n_{i}}\left(A_{i}\right)$, the $c_{0}$-sum of $M_{n_{i}}\left(A_{i}\right)$, where $I$ is an arbitrary index set, and for every $i \in I, M_{n_{i}}\left(A_{i}\right)$ stands for the $n_{i} \times n_{i}$ matrices with entries in a commutative $C^{*}$-algebra $A_{i}$. Moreover our theorem applies to group $C^{*}$-algebras of compact groups. In fact we show that every multilinear form on $\bigoplus_{i \in I} C\left(\Omega_{i}, M_{n_{i}}\right)$, where $\Omega_{i}$ is a scattered topological space for each $i \in I$, can be approximated by norm attaining multilinear forms.

THEOREm 3. Let $\left(n_{i}\right)_{i \in I}$ be a family of positive integers, let $\left(\Omega_{i}\right)_{i \in I}$ be a family of scattered locally compact spaces, and let $A=\bigoplus_{i \in I} C\left(\Omega_{i}, M_{n_{i}}\right)$. 
Then every continuous multilinear form on A can be approximated by norm attaining multilinear forms.

\section{Proofs}

Proof of Theorem 1. A careful analysis of the proof of [16, Theorem B] (see [4] for the complex version of this proof) shows the first assertion of Theorem 1 . The second assertion follows by the same method as in $[4$, Theorem 3].

Proof of Theorem 2. We begin with a simple but useful observation that will be frequently used throughout the proof. If $\mu \in A^{*}, x \in A^{* *}$, and $z \in A^{* *}$ is a central projection, then

$$
|\mu(z x)| \leq|\mu|(z)\|x\|,
$$

where, from now on, $|\mu|$ stands for the absolute value of $\mu$. Indeed, let $u$ be a partial isometry such that $\mu(y)=|\mu|(u y)$ for each $y \in A^{* *}$. Since the linear functional $\lambda$ on $A^{* *}$ defined by $\lambda(y)=|\mu|(z y)$ for each $y \in A^{* *}$ is positive it follows that it attains its norm at $\mathbf{1}$. We thus get

$$
|\mu(z x)|=|| \mu|(z u x)|=|\lambda(u x)| \leq \lambda(\mathbf{1})\|u x\|=|\mu|(z)\|u x\| \leq|\mu|(z)\|x\| .
$$

We now turn to the proof of the theorem. On account of Theorem 1 together with the result in [2] that every continuous linear operator from $A$ into $A^{*}$ is weakly compact, we only need to show that for every weakly compact subset $W \subset A^{*}$, every $\mu_{0} \in A^{*}$, and every $\varepsilon>0$ there exists a continuous linear operator $S: A^{*} \rightarrow A^{*}$ with the properties required in Theorem 1.

In case $\mu_{0}=0$ we just take $S$ to be the identity operator on $A^{*}$, so we assume $\mu_{0} \neq 0$. Moreover by replacing $W$ with $W \cup\left\{\mu_{0}\right\}$ we may assume that $\mu_{0} \in W$. Since $W$ is weakly compact, [17, Theorem III.5.iv] shows that there exist a state $\phi$ on $A$ and $\delta>0$ with the following property:

$$
|\mu(x)|<\min \left\{\varepsilon / 2,\left\|\mu_{0}\right\|\right\} \text { for all } \mu \in W \text { and } x \in A^{* *} \text { such that }\|x\| \leq 1
$$
and $\phi\left(x x^{*}+x^{*} x\right)<\delta$.

On account of $(\mathrm{H})$, there exist a positive linear functional $\psi$ on $A$ and $\alpha>0$ with the following property:

(2) If $p \in A^{* *}$ is a projection with $\psi(p)<\alpha$ then there exists a central projection $z \in A^{* *}$ such that $z p=0$ and $\phi(1-z)<\delta / 4$.

Let $u \in A^{* *}$ be a partial isometry such that $\left\|\mu_{0}\right\|=\mu_{0}(u)$. According to Lusin's Theorem [17, Theorem II.4.15], there exist $a \in A$ and a projection $p \in A^{* *}$ such that

$$
\|a\|<1+\alpha, \quad \text { ap }=u p, \quad \psi(\mathbf{1}-p)<\alpha .
$$


From (2) it may be concluded that there exists a central projection $z \in A^{* *}$ such that

$$
z p=z \mathbf{1}=z, \quad \phi(\mathbf{1}-z)<\delta / 4 .
$$

Note that

$$
a z=u z .
$$

We now proceed to show that there exist $a_{0} \in A$ and a central projection $w \in A^{* *}$ such that

$$
\left\|a_{0}\right\|=1, \quad a_{0} w=u w, \quad \phi(\mathbf{1}-w)<\delta / 2 .
$$

To this end, consider the $C^{*}$-subalgebra $B$ of $A$ generated by $a$ and $a^{*}$ and let $B^{\mathrm{cc}}$ be its bicommutant in $A^{* *}$. Since $z \in B^{\mathrm{cc}}$ and $B$ is separable, [18, Theorem 5] gives a projection $q \in B^{\mathrm{cc}}$ which is regular relative to $B$ and such that $q \leq z$ and $\psi(z-q)<\alpha$. Since $q \leq z$, it follows that $a q=u q$. Let $\bar{q}$ denote the closure of $q$ in $B^{\text {cc }}$. The regularity of $q$ together with [3, Proposition II.12] yields $\|a \bar{q}\|=\|a q\|$, so $\|a \bar{q}\| \leq 1$. Now [7, Theorem 3.3(a)] shows that there exist $b \in B$ such that $b \bar{q}=0$ and $\|a-b\| \leq 1$. Since $q \leq \bar{q}$, it follows that $b q=0$ and therefore that $(a-b) q=u q$. We take $a_{0}=a-b$. Since $\psi(z-q)<\alpha,(2)$ shows that there exists a central projection $v \in A^{* *}$ such that $v z=v q$ and $\phi(\mathbf{1}-v)<\delta / 4$. We take $w=v z$. It is easily seen that $a_{0} w=u w$. On the other hand, we have

$$
\phi(\mathbf{1}-w)=\phi(\mathbf{1}-z)+\phi(z(\mathbf{1}-v)) \leq \phi(\mathbf{1}-z)+\phi(\mathbf{1}-v)<\delta / 2 .
$$

Since $u w$ is a partial isometry, it follows that either $\|u w\|=1$ or $u w=0$. We claim that $\|u w\|=1$. We have $\left\|\mu_{0}\right\|=\mu_{0}(u)$. Since $\|u(\mathbf{1}-w)\| \leq 1$ and

$$
\begin{aligned}
\phi\left((u(\mathbf{1}-w))^{*}(u(\mathbf{1}-w))\right. & \left.+(u(\mathbf{1}-w))(u(\mathbf{1}-w))^{*}\right) \\
& =\phi\left(\left(u^{*} u+u u^{*}\right)(\mathbf{1}-w)\right) \leq 2 \phi(\mathbf{1}-w)<\delta,
\end{aligned}
$$

(1) shows that $\mu_{0}(u(\mathbf{1}-w))<\left\|\mu_{0}\right\|=\mu_{0}(u)$ and so $u w \neq 0$. We thus get $\left\|a_{0} w\right\|=1$, and because $\left\|a_{0}\right\| \leq 1$, this clearly forces $\left\|a_{0}\right\|=1$ as required.

We are now in a position to define the desired operator. Let $\gamma$ be a state of $A$ such that $\gamma\left(a_{0}\right)=1$ and let $S: A^{*} \rightarrow A^{*}$ be the continuous linear operator defined by

$$
(S \mu)(y)=\mu(w y)+\mu((\mathbf{1}-w) u) \gamma(y)
$$

for all $\mu \in A^{*}$ and $y \in A$. Since

$$
|(S \mu)(y)| \leq|\mu(w y)|+|\mu((\mathbf{1}-w) u)| \leq|\mu|(w)+|\mu|(\mathbf{1}-w)=|\mu|(\mathbf{1})=\|\mu\|
$$

for each $y \in A$ such that $\|y\|=1$, we conclude that $\|S\| \leq 1$. Furthermore, we have

$$
\begin{aligned}
\left(S \mu_{0}\right)\left(a_{0}\right) & =\mu_{0}\left(w a_{0}\right)+\mu_{0}((\mathbf{1}-w) u) \gamma\left(a_{0}\right) \\
& =\mu_{0}(w u)+\mu_{0}((\mathbf{1}-w) u)=\mu_{0}(u)=\left\|\mu_{0}\right\|
\end{aligned}
$$


Finally, if $x \in A^{* *}$ is such that $\|x\|=1$, then $\|(\mathbf{1}-w) x\| \leq 1$ and

$$
\begin{aligned}
\phi\left(((\mathbf{1}-w) x)^{*}((\mathbf{1}-w) x)+\right. & \left.((\mathbf{1}-w) x)((\mathbf{1}-w) x)^{*}\right) \\
& =\phi\left((\mathbf{1}-w)\left(x^{*} x+x x^{*}\right)\right) \leq 2 \phi(\mathbf{1}-w)<\delta .
\end{aligned}
$$

On account of (1), we have $\mu((\mathbf{1}-w) x)<\varepsilon / 2$ for each $\mu \in W$. Therefore

$$
|(S \mu-\mu)(a)| \leq|\mu((\mathbf{1}-w) a)|+|\mu((\mathbf{1}-w) u)|<\varepsilon
$$

for each $a \in A$ such that $\|a\|=1$ and for each $\mu \in W$. Thus $\|S \mu-\mu\|<\varepsilon$, for all $\mu \in W$, which completes the proof.

REMARK 1. Under the hypothesis $(\mathrm{H})$ of Theorem 2, it is worth pointing out that every weakly compact operator from $A_{\text {sa }}$ into an arbitrary Banach space can be approximated by norm attaining operators and that every continuous bilinear form on $A_{\text {sa }}$ can be approximated by norm attaining bilinear forms on $A_{\mathrm{sa}}$. This follows by the same method as in the preceding proof (taking into account the identification of $\left(A_{\mathrm{sa}}\right)^{*}$ with $\left.\left(A^{*}\right)_{\mathrm{sa}}\right)$. It suffices to observe that in such a situation $u \in A_{\text {sa }}$ and that therefore we can replace $a_{0}$ by $b_{0}=\frac{1}{2}\left(a_{0}+a_{0}^{*}\right)$.

Proof of Theorem 3. First, let us note that $A^{*}=\ell_{1}-\bigoplus_{i \in I} C_{0}\left(\Omega_{i}, M_{n_{i}}\right)^{*}$. Since $\Omega_{i}$ is scattered for each $i \in I$, it follows that there exists a set $J_{i}$ such that $C_{0}\left(\Omega_{i}, M_{n_{i}}\right)^{*}\left(=M\left(\Omega_{i}, M_{n_{i}}^{*}\right)\right)$ can be identified with $\ell_{1}-\bigoplus_{j \in J_{i}} M_{n_{i}}^{*}$. Thus

$$
A^{*}=\ell_{1}-\bigoplus_{\lambda \in \Lambda} M_{n_{\lambda}}^{*},
$$

where $\Lambda=\bigcup_{i \in I} I \times J_{i}$ and $n_{\left(i, j_{i}\right)}=n_{i}$ for each $\left(i, j_{i}\right) \in \Lambda$.

Now we claim that $A^{*}$ has the Schur property. Let $\left(\phi_{n}\right)$ be a sequence in $A^{*}$ which converges weakly to zero. Then there exists a countable set $\Lambda_{1} \subset \Lambda$ such that $\left(\phi_{n}\right)$ lies in $\ell_{1}-\bigoplus_{\lambda \in \Lambda_{1}} M_{n_{\lambda}}^{*}$. On account of [6, Corollary 2.6], $\ell_{1}-\bigoplus_{\lambda \in \Lambda_{1}} M_{n_{\lambda}}^{*}$ has the Schur property and therefore $\lim \left\|\phi_{n}\right\|=0$, which proves our claim.

Now we prove that for every $N \in \mathbb{N}, \mathcal{L}\left({ }^{N} A\right)$ does not contain $c_{0}$ and every continuous linear operator from $A$ into $\mathcal{L}\left({ }^{N} A\right)$ is compact, where $\mathcal{L}\left({ }^{N} A\right)$ stands for the Banach space of all continuous $N$-linear forms on $A$.

If $N=1$, then $\mathcal{L}\left({ }^{N} A\right)$ becomes $A^{*}$, which has the Schur property and so does not contain $c_{0}$. On the other hand every continuous linear operator from $A$ into $A^{*}$ is weakly compact ([2]) and therefore it is compact, again by the Schur property.

Assume by induction that $\mathcal{L}\left({ }^{N} A\right)$ does not contain $c_{0}$ and that every operator from $A$ into $\mathcal{L}\left({ }^{N} A\right)$ is compact. Since $A$ does not contain $\ell_{1}$ and $\mathcal{L}\left({ }^{N} A\right)$ does not contain $\ell_{\infty}, \mathcal{L}\left({ }^{N+1} A\right)=\mathcal{L}\left(A, \mathcal{L}\left({ }^{N} A\right)\right)$ does not contain $\ell_{\infty}$ (by [14, Theorem 4]) and therefore it cannot contain $c_{0}$, since $\mathcal{L}\left({ }^{N+1} A\right)$ is a dual space. Now we can use [2] to deduce that every operator from $A$ 
into $\mathcal{L}\left({ }^{N+1} A\right)$ is weakly compact. Since $A^{*}$ has the Schur property, $A$ has the Dunford-Pettis property and by the Brace-Grothendieck Theorem (see $[10$, p. 177]), every such operator takes weakly Cauchy sequences into norm convergent sequences. Since $A$ does not contain $\ell_{1}$, Rosenthal's $\ell_{1}$-theorem establishes the required property.

Now we are in a position to prove the theorem. It is easy to check that every finite linear combination of $N$-linear forms on $A$ of the type

$$
B\left(a_{1}, \ldots, a_{N}\right)=\prod_{j=1}^{N} \xi_{j}\left(a_{j}\left(i_{j}\right)\left(t_{j}\right)\right),
$$

where $i_{j} \in I, t_{j} \in \Omega_{i_{j}}$ and $\xi_{i} \in M_{n_{i_{j}}}^{*}$ for $j=1, \ldots, N$, attains its norm. Denote by $\mathcal{F}_{N}$ the linear span of the $N$-linear forms of the above type. We are going to prove that $\mathcal{F}_{N}$ is dense in $\mathcal{L}\left({ }^{N} A\right)$ for each $N \in \mathbb{N}$. The case $N=1$ is immediate. Assume that $\mathcal{F}_{j}$ is dense in $\mathcal{L}\left({ }^{j} A\right)$ for $j \leq N$. Let $\Phi$ be an operator from $A$ into $\mathcal{L}\left({ }^{N} A\right)$. Since $\Phi$ is compact and $A$ has the approximation property, it can be approximated by a finite rank operator $\Psi$. Therefore there exist $\phi_{1}, \ldots, \phi_{p} \in A^{*}$ and $\left.\Psi_{1}, \ldots, \Psi_{p} \in \mathcal{L}\left({ }^{N} A\right)\right)$ such that

$$
\Psi(a)=\sum_{j=1}^{p} \phi_{j}(a) \Psi_{j} \quad \text { for each } a \in A .
$$

Since $\mathcal{F}_{N}$ is dense in $\mathcal{L}\left({ }^{N} A\right)$ and $\mathcal{F}_{1}$ is dense in $A^{*}$, it may be concluded that $\Psi$ can be approximated by an element of $\mathcal{F}_{N+1}$.

\section{Examples}

Lemma 1. Let $A$ be a commutative $C^{*}$-algebra and let $n \in \mathbb{N}$. Then the $C^{*}$-algebra $M_{n}(A)$ satisfies $(\mathrm{H})$.

Proof. We can certainly assume that $A$ is the $C^{*}$-algebra $C_{0}(\Omega)$ of all complex-valued continuous functions on a locally compact Hausdorff space $\Omega$ vanishing at infinity so that $M_{n}(A)$ is identified with $C_{0}\left(\Omega, M_{n}(\mathbb{C})\right)$. On the other hand, $M_{n}(A)^{*}$ is identified with $M_{n}(\mathcal{M}(\Omega))$ and $M_{n}(A)^{* *}$ is identified with the von Neumann algebra $M_{n}\left(\mathcal{M}(\Omega)^{*}\right)$, where $\mathcal{M}(\Omega)$ is the Banach space of all complex-valued regular Borel measures on $\Omega$.

Let $\phi=\left(\phi_{i j}\right)$ be a positive linear functional on $M_{n}(A)$ and $\varepsilon>0$. Then $\phi_{i i}$ is a positive measure on $\Omega$ for each $i=1, \ldots, n$ and we consider the measure $\lambda=\sum_{i=1}^{n} \phi_{i i}$. Let tr denote the usual trace on $M_{n}(\mathbb{C})$, let $\psi$ be the positive linear functional on $M_{n}(A)$ defined by

$$
\psi(a)=\int_{\Omega} \operatorname{tr}(a(t)) d \lambda(t)
$$

for each $a \in M_{n}(A)$, and let $\delta=\varepsilon$. 
Suppose that $p \in M_{n}(A)^{* *}$ is a projection such that $\psi(p)<\delta$. Then $p$ can be thought of as a function in $L^{\infty}\left(\lambda, M_{n}(\mathbb{C})\right)$ such that $p(t)$ is a projection in $M_{n}(\mathbb{C})$ for each $t \in \Omega$. Set

$$
\Delta=\{t \in \Omega: p(t) \neq 0\} .
$$

Of course $\operatorname{tr}(p(t)) \geq 1$ for $t \in \Delta$ and therefore

$$
\lambda(\Delta)=\int_{\Delta} d \lambda(t) \leq \int_{\Delta} \operatorname{tr}(p(t)) d \lambda(t) \leq \psi(p)<\varepsilon,
$$

which gives $\lambda(\Delta)<\varepsilon$. The central projection $z \in M_{n}(A)^{* *}$ given by $z(t)=$ $0 \in M_{n}(\mathbb{C})$ if $t \in \Delta$ and $z(t)=\mathbf{1} \in M_{n}(\mathbb{C})$ elsewhere clearly satisfies $z p=0$. Moreover $\phi(\mathbf{1}-z)=\sum_{i=1}^{n} \phi_{i i}(\Delta)=\lambda(\Delta)<\varepsilon$, as required.

Lemma 2. Let $\left(A_{i}\right)_{i \in I}$ be a family of $C^{*}$-algebras satisfying $(\mathrm{H})$. Then $A=\bigoplus_{i \in I} A_{i}$ satisfies $(\mathrm{H})$.

Proof. Let $\phi$ be a positive linear functional on $A$ and $\varepsilon>0$. We have to prove that there exist $\psi \in A_{+}^{*}$ and $\delta>0$ satisfying the conditions of property $(\mathrm{H})$.

Since $A^{*}=\ell_{1}-\bigoplus_{i \in I} A_{i}^{*}$, it follows that $\phi=\left(\phi_{i}\right)$ with $\phi_{i} \in A_{i}^{*}$ for each $i \in I$ and $\|\phi\|=\sum_{i \in I}\left\|\phi_{i}\right\|$. Then there exists a finite subset $F$ of $I$ such that $\sum_{i \in F}\left\|\phi_{i}\right\|>\|\phi\|-\varepsilon / 2$.

Now, we can apply the assumption that property $(\mathrm{H})$ holds for $A_{i}$, and so there exist $\delta_{i}>0$ and positive linear functionals $\psi_{i}$ on $A_{i}$ such that if $p_{i} \in A_{i}^{* *}$ is a projection with $\psi_{i}\left(p_{i}\right)<\delta_{i}$ then there exists a central projection $z_{i} \in A_{i}^{* *}$ such that

$$
z_{i} p_{i}=0, \quad \phi_{i}\left(\mathbf{1}-z_{i}\right)<\frac{\varepsilon}{2 \operatorname{card}(F)} .
$$

Let $\delta=\min \left\{\delta_{i}: i \in F\right\}$ and $\psi=\left(\theta_{i}\right)_{i \in I}$, where $\theta_{i}=\psi_{i}$ if $i \in F$ and $\theta_{i}=0$ otherwise.

Let $p$ be a projection in $A^{* *}$ such that $\psi(p)<\delta$. Since $A^{* *}=\ell_{\infty^{-}}$ $\bigoplus_{i \in I} A_{i}^{* *}$, it follows that $p=\left(p_{i}\right)$ with $p_{i} \in A_{i}^{* *}$ a projection. On the other hand, $\psi_{i}\left(p_{i}\right)<\delta \leq \delta_{i}$ for each $i \in F$. Therefore we can find a central projection $z_{i} \in A_{i}^{* *}$ such that

$$
z_{i} p_{i}=0, \quad \phi_{i}\left(\mathbf{1}-z_{i}\right)<\varepsilon /(2 \operatorname{card}(F)), \quad \forall i \in F .
$$

Then the central projection

$$
z= \begin{cases}z_{i} & \text { if } i \in F \\ 0 & \text { if } i \notin F\end{cases}
$$

satisfies $z p=0$ and 


$$
\begin{aligned}
\phi(\mathbf{1}-z) & =\sum_{i \in F} \phi_{i}\left(\mathbf{1}-z_{i}\right)+\sum_{i \notin F} \phi_{i}(\mathbf{1}) \\
& =\sum_{i \in F} \phi_{i}\left(\mathbf{1}-z_{i}\right)+\sum_{i \notin F}\left\|\phi_{i}\right\|<\frac{\operatorname{card}(F) \varepsilon}{2 \operatorname{card}(F)}+\frac{\varepsilon}{2}=\varepsilon
\end{aligned}
$$

Recall that the group $C^{*}$-algebra $C^{*}(G)$ of a locally compact group $G$ is the completion of $L^{1}(G)$ with respect to the norm given by

$\|f\|=\sup \{\|\pi(f)\|: \pi$ is an irreducible unitary representation of $G\}$.

Corollary 4. Let $H$ be a locally compact abelian group and let $K$ be a compact group. Then the following assertions hold:

(1) There exists a family $\left(n_{i}\right)_{i \in I}$ of positive integers such that $C^{*}(K)$ is *-isomorphic to $\bigoplus_{i \in I} M_{n_{i}}$.

(2) $C^{*}(H \times K)$ is *-isomorphic to $\bigoplus_{i \in I} C_{0}\left(\widehat{H}, M_{n_{i}}\right)$, where $\widehat{H}$ is the dual group of $H$.

Accordingly, every continuous multilinear form on $C^{*}(K)$ can be approximated by norm attaining multilinear forms and every bilinear form on $C^{*}(H \times K)$ can be approximated by norm attaining bilinear forms.

Proof. (1) On account of [12, Theorem 28.40], $L^{1}(K)$ can be embedded as a dense subalgebra of $\bigoplus_{[\sigma] \in \widehat{K}} M_{n_{\sigma}}$, where here and subsequently $\widehat{K}$ stands for the dual object of $K$ and $[\sigma]$ stands for the equivalence class of an irreducible unitary representation $\sigma$ of $K$. Consequently, $C^{*}(K)=\bigoplus_{[\sigma] \in \widehat{K}} M_{n_{\sigma}}$. On account of Theorem 3 every multilinear form on $C^{*}(K)$ can be approximated by norm attaining multilinear forms.

(2) We define $\Phi: L^{1}(H) \otimes L^{1}(K) \rightarrow C_{0}\left(\widehat{H}, C^{*}(K)\right)$ by

$$
\Phi(f \otimes g)(\gamma)=\widehat{f}(\gamma) g
$$

for all $f \in L^{1}(H), g \in L^{1}(K)$, where $\widehat{f}$ denotes the Fourier transform of $f$.

Let us check that $\Phi$ preserves norms. Take $F=\sum_{i=1}^{n} f_{i} \otimes g_{i}$ in $L^{1}(G) \otimes$ $L^{1}(H)$. Then

$$
\begin{aligned}
\|\Phi(F)\|_{\infty} & =\sup _{\gamma \in \widehat{H}} \sup _{\sigma \in \widehat{K}}\|\sigma(\Phi(F)(\gamma))\| \\
& =\sup _{\gamma \in \widehat{H}} \sup _{\sigma \in \widehat{K}}\left\|\sum_{i=1}^{n} \widehat{f}_{i}(\gamma) \sigma\left(g_{i}\right)\right\|=\|F\|,
\end{aligned}
$$

because every irreducible unitary representation $\pi$ of $H \times K$ is of the form $\tau \otimes \sigma$, where $\tau$ and $\sigma$ are irreducible unitary representations of $H$ and $K$ respectively (see [15, Section 12.4.30]). Since $L^{1}(H) \times L^{1}(K)$ is dense in $L^{1}(H \times K)$ with respect to $\|\cdot\|_{1}$, and $\|\cdot\| \leq\|\cdot\|_{1}$, it follows that $L^{1}(H) \otimes L^{1}(K)$ 
is dense in $C^{*}(H \times K)$. On the other hand,

$$
\Phi\left(L^{1}(H) \otimes L^{1}(K)\right)=A(\widehat{H}) \otimes L^{1}(K),
$$

where $A(\widehat{H})$ denotes the Fourier algebra of $\widehat{H}$. Since $A(\widehat{H})$ is dense in $C_{0}(\widehat{H})$ and $L^{1}(K)$ is dense in $C^{*}(K)$, it may be concluded that $\Phi\left(L^{1}(H) \otimes L^{1}(K)\right)$ is dense in $C_{0}\left(\widehat{H}, C^{*}(K)\right)$. Therefore $\Phi$ extends to a $*$-isomorphism from $C^{*}(H \times K)$ onto $C_{0}\left(\widehat{H}, C^{*}(K)\right)$.

Finally, since $C^{*}(K) \equiv \bigoplus_{i \in I} M_{n_{i}}$, it follows that

$$
C_{0}\left(\widehat{H}, C^{*}(K)\right) \equiv \bigoplus_{i \in I} C_{0}\left(\widehat{H}, M_{n_{i}}\right) \text {. }
$$

According to Lemmas 1 and $2, C^{*}(H \times K)$ has property $(\mathrm{H})$ and Theorem 2 now shows that every bilinear form on $C^{*}(H \times K)$ can be approximated by norm attaining bilinear forms.

REMARK 2. It is worth pointing out that by the Freudenthal-Weil Theorem's [15, Theorem 12.4.28], every connected Moore group $G$ can be written as a product of a vector group and a compact group and therefore Corollary $4(2)$ holds true for such a group.

\section{References}

[1] M. D. Acosta, F. J. Aguirre and R. Payá, There is no bilinear Bishop-Phelps theorem, Israel J. Math. 93 (1996), 221-227.

[2] C. A. Akemann, The dual space of an operator algebra, Trans. Amer. Math. Soc. 126 (1967), 286-203.

[3] —, The general Stone-Weierstrass problem, J. Funct. Anal. 4 (1969), 277-294.

[4] J. Alaminos, Y. S. Choi, S. G. Kim, and R. Payá, Norm attaining bilinear forms on spaces of continuous functions, Glasgow Math. J. 40 (1998), 359-365.

[5] R. M. Aron, C. Finet, and E. Werner, Some remarks on norm-attaining n-linear forms, in: Function Spaces (Edwardsville, IL, 1994), K. Jarosz (ed.), Lecture Notes in Pure and Appl. Math. 172, Marcel Dekker, 1995, 19-28.

[6] J. Bourgain, New Classes of $\mathcal{L}^{p}$-spaces, Lecture Notes in Math. 889, Springer, 1981.

[7] L. G. Brown, Semicontinuity and multipliers of $C^{*}$-algebras, Canad. J. Math. 40 (1988), 865-988.

[8] Y. S. Choi, Norm attaining bilinear forms on $L^{1}[0,1]$, J. Math. Anal. Appl. 211 (1997), 295-300.

[9] Y. S. Choi and S. G. Kim, Norm or numerical radius attaining multilinear mappings and polynomials, J. London Math. Soc. 54 (1996), 135-147.

[10] J. Diestel and J. J. Uhl Jr., Vector Measures, Math. Surveys 15, Amer. Math. Soc., 1977.

[11] C. Finet and R. Payá, Norm attaining operators from $L_{1}$ into $L_{\infty}$, Israel J. Math. 108 (1998), 139-143.

[12] E. Hewitt and K. A. Ross, Abstract Harmonic Analysis II, Springer, 1970.

[13] M. Jiménez-Sevilla and R. Payá, Norm attaining multilinear forms and polynomials on preduals of Lorentz sequence spaces, Studia Math. 127 (1998), 407-418. 
[14] N. Kalton, Spaces of compact operators, Math. Ann. 208 (1974), 267-278.

[15] T. W. Palmer, Banach Algebras and the General Theory of *-Algebras, Vol. II, Encyclopedia Math. Appl. 79, Cambridge Univ. Press, 2001.

[16] W. Schachermayer, Norm attaining operators on some classical Banach spaces, Pacific J. Math. 105 (1983), 427-438.

[17] M. Takesaki, Theory of Operator Algebras I, Springer, 1979.

[18] M. Tomita, Spectral theory of operator algebras I, Math. J. Okayama Univ. 9 (1959), 63-98.

Departamento de Análisis Matemático

Facultad de Ciencias

Universidad de Granada

18071 Granada, Spain

E-mail: alaminos@ugr.es

rpaya@ugr.es

avillena@ugr.es

Received January 25, 2002

Revised version December 18, 2002 